\title{
Measurement of environmental pollutants using passive sampling devices - an updated commentary on the current state of the art
}

\section{Abstract}

The following provides a short overview of the important topics arising from the 6th International Passive Sampling Workshop and Symposium (IPSW 2013) held in Bordeaux, France between 26-29th June, 2013. Most of the discussions focussed on monitoring non-polar and polar organic pollutants in water with less coverage on air (probably already seen as a mature technology for this medium) and sediments. The use of passive sampling devices within regulatory water monitoring programmes was also a major theme of the Workshop.

A number of passive samplers have been available for over forty years to measure chemicals in different environmental media (e.g. air, soils, sediments and water) ${ }^{1}$. The technique can be used to measure either equilibrium or time-weighted average concentrations (TWA) of the analyte of concern. Historically, such devices have been used to monitor localised ambient work place chemicals or atmospheric pollutants on a global scale (e.g. within the United Nations Stockholm Convention on the transboundary movement of persistent organic pollutants using large networks of samplers). The use of passive samplers for monitoring pollutants in sediments, soils and water is a more recent development, but one that is gathering momentum internationally. It is now recognised that these devices can have important roles in monitoring water quality across the European Community within the remit of various legislative (e.g. Water Framework Directive (WFD) and Marine Strategy Framework Directive (MSFD)) regulations ${ }^{2-4}$. Typically, data obtained from samplers can be used alongside information obtained from conventional spot sampling of water to assist in checking compliance with environmental quality criteria or for assessment of longterm pollution trends. Use of this combined approach helps to improve risk assessments and to better inform decisions on undertaking potentially expensive remedial actions. Devices can also be used for sampling of more complex environmental matrices such as sediments and to mimic the uptake of chemicals by biota. For example, the measurement of the freely dissolved concentration of a chemical in pore waters of sediments and soils as well as its accessible (releasable) 
concentration from these media, are important parameters in environmental risk assessments.

At the $6^{\text {th }}$ International Passive Sampling Workshop and Symposium (IPSW 2013) held in Bordeaux, France between 26-29 ${ }^{\text {th }}$ June, 2013 (the previous European events took place in the Czech Republic in 2004 and 2009, Slovakia in 2006 and Poland in 2011) a number of important developments and the future challenges in the use of passive sampling technology were discussed. The event was attended by over 70 delegates from 17 countries and provided a timely opportunity for international experts to discuss key research and regulatory issues. The following article provides an update of the important topics arising from the symposium since the last commentary published in $2011^{5}$; most being centred on monitoring organic pollutants in water with less coverage on air (probably already seen as a mature technology for this sector) and sediments.

\section{Measuring concentrations of non-polar pollutants in water}

The use of passive samplers to monitor non-polar chemicals (e.g. PAHs, PCBs, chlorinated pesticides and industrial chemicals) in water was the first application of such devices for this medium. In the 1990s, nearly all trials used the semi-permeable membrane device (SPMD): an enclosed low-density polyethylene (LDPE) membrane filled with a small amount of the lipid triolein as receiving phase ${ }^{6}$. With this sampler and with these classes of pollutants, performance reference compounds (PRCs) can be used for in-situ calibration. PRCs reduce the uncertainty of the TWA concentration data produced during field deployments where changes in the water temperature and turbulence can affect the sampler uptake rates (usually measured in L/day). Over the last ten years there has been a move away from SPMDs to using low-cost single-phase polymers such as low-density polyethylene (LDPE) and silicone rubber ${ }^{7}$. Such materials are flexible as they can be fashioned to any size or thickness for varying field applications. Robust cleaning procedures are available to remove contaminant chemicals or residual monomers from these materials. In addition, their extraction and clean-up procedures are relatively simple in comparison to those needed for SPMDs. Robust calibration procedures (to measure the sampler uptake rate and the sample/water partition coefficient $\left(K_{s w}\right)$ for different chemicals) are in place for single-phase samplers and hence these can be used with confidence 
in regulatory monitoring programmes. Other polymers may be used for niche applications (e.g. polyimide, polyoxymethylene, polypropylene,) and to cover a wider range of pollutant classes. The use of specific physico-chemical properties (e.g. Hansen solubility parameter) of an analyte and matching these to the chemistry of a specific polymer was highlighted as a way to aid in material selection and for modelling purposes. This work is being undertaken by the Safety and Environmental Assurance Centre within Unilever, UK. Application of single-phase samplers to monitor specific classes of emerging pollutants such as methyl siloxanes and organo-tins were presented at the meeting. As their dissolved concentrations are very low ( $\mathrm{ng} / \mathrm{L}$ or sub $\mathrm{ng} / \mathrm{L}$ ) this is often below the detection limit of analytical instrumentation used to measure these compounds; the use of passive samplers offers a significant advantage over spot sampling approaches.

Another application is to use such polymers (typically silicone rubber) deployed on research ships and ferries. Rubber samplers were used by Cefas (Lowestoft Laboratory, UK) housed within their research vessel RV Endeavour. Sea water was pumped across the devices (contained in a special box) in a controlled way to minimise flow effects on sampler uptake rate for the target pollutants. Information on the spatial distribution of pollutants could be obtained and at a lower cost compared with conventional means of collecting such data. This approach was also used on Joint Danube Survey (JDS3: http://danubesurvey.org/) where an "active" passive sampler system was installed on board the expedition ship. Water sampling took place over a series of 5-day intervals as the vessel moved downstream along defined stretches of the river. High sampler uptake rates were achieved with subsequent enhanced analytical detection limits for chemicals. This temporally- and spatially-integrative sampling approach provides representative information on water quality over defined stretches of the Danube. Samplers can also be deployed easily on gliders and other remotely controlled apparatus used in oceanographic surveys and can potentially give data on concentrations of pollutants with water depth as well as spatially.

\section{Measuring concentrations of polar pollutants in water}


Over the last five years there has been increased interest in measuring the concentration of a range of polar chemicals in water ${ }^{8}$. Many of these substances are classified as 'emerging pollutants'. Two designs of sampler are generally used, the polar organic compound integrative sampler (POCIS) and the polar version of the Chemcatcher $^{\circledR}$. Most published work used the POCIS. Both devices use receiving phases that sequester polar pollutants by an adsorption or ion-exchange mechanism rather than by partition and both use a thin protective polyethersulphone (PES) diffusion membrane. Typically, sampler uptake rates are 10-250 mL/day for polar compounds. Uptake for most analytes remains in the linear phase over about a 14day period with most chemicals exhibiting only a short lag time. The mechanism of uptake for polar compounds is not well understood, particularly the transfer kinetics of chemicals across the PES membrane and this is an area for further research. Changing the type of diffusion membrane (e.g. Nylon) to decrease lag-phase phenomenon and to improve uptake kinetics has been proposed.

A further drawback is that there is a lack of theoretical models able to predict the uptake of a chemical into a POCIS or Chemcatcher ${ }^{\circledR}$ based on the compounds physicochemical properties (e.g. $\log K_{\text {ow }}$ ). Hence, this necessitates extensive laboratory-based calibration experiments to measure compound specific uptake rates (and in some case the effects of temperature, turbulence and salinity) before the samplers can be used in the field to measure TWA concentrations ${ }^{9}$. The use of PRCs with adsorption or ion-exchange based systems is still not fully demonstrated, although some groups have shown that pre-loading the receiving phase with deuterated $\left(d_{5}\right)$ deisopropylatrazine can possibly be used for this purpose ${ }^{10}$. These factors thus limit the utility of these samplers beyond screening or semi-quantitative assessment of pollutants. The development of an organic version (o-DGT) of the diffusion in thin films (DGT) device used for metals is however showing some promise $^{11}$. Here a thick diffusion gel layer is added, which helps control the uptake of analytes into the receiving phase and limits the effects of water flow. This may address the problem of the lack of a PRC approach for the polar Chemcatcher ${ }^{\circledR}$ and POCIS samplers.

Several groups questioned the effect of uneven distribution of the loose sorbent within POCIS, which can sag towards the base of the device during extended 
deployments, potentially reducing the active sampling surface area. This issue may be resolved easily by directly replacing the powder by a commercially available 47 mm extraction disk (e.g. 3M Empore ${ }^{\mathrm{TM}}$ or Horizon Technology Inc. Atlantic ${ }^{\circledR}$ disks) that is available for a range of chemistries. Using such a simple design modification should help minimise variability of field data. Natural Resources Wales are starting to deploy this new design of sampler in effluents at waste water treatment plants; initial results for the screening of pharmaceuticals using such devices and liquid chromatography with time-of-flight mass spectrometry detection techniques are encouraging.

Applications of polar samplers to measure pharmaceuticals, personal care products and other chemicals (e.g. polar pesticides, acid herbicides, alkylphenols) in various aquatic matrices (e.g. drinking, surface and waste water and hospital effluents) were discussed. As there is a paucity of reliable uptake rates available in the literature for polar compounds, when quantitative results are required an extensive laboratory calibration step is required. No standard calibration (e.g. using static, semi-static, or through-flow tanks) procedure is being used among practitioners and this naturally increases the variability of results. In addition, the aqueous matrix used for calibration can have a significant impact on the value of the sampling rate obtained. For example sampling rates are known to be different when measured in laboratorygrade distilled water compared with those obtained using a waste water effluent ${ }^{12}$. A novel approach is to use in situ field calibrations in order to obtain sampler uptake rates and this is particularly suited for hydrophilic chemicals. If the field concentration of a substance is known to be relatively constant (the concentration usually first established by the intensive collection of water samples over an extended period of time) then in situ calibration is a possibility. It is useful for substances such as human metabolites of pharmaceuticals that are difficult and expensive to obtain in sufficient quantities needed for laboratory tests. Typically samplers can be deployed in the influent or effluent of a well controlled waste water treatment plant to obtain such calibration data. In situ calibration may also be attractive in other complex matrices such as estuarine, halo-saline and marine environments where salinity may influence uptake kinetics. 
In future better guidance on the range of approaches for the calibration of samplers is needed, particularly if devices are to be incorporated into large scale monitoring programmes. Such a document would be a useful adjunct to the ISO standard: Water quality - Sampling Part 23: Guidance on passive sampling in surface waters (ISO 5667-23:2011). This was designed to help standardise the application of different passive samplers by end users and thus to facilitate the use of this technology within a regulatory monitoring framework.

\section{Measuring concentrations of metals and other inorganic compounds in water}

Passive samplers have been used to monitor metals and other inorganic compounds in water for many years. Most work uses the DGT device and sometimes the metals version of the Chemcatcher ${ }^{\circledR 13}$. Often devices are used alongside other types of samplers to monitor a wide suite of pollutants in the water column. In addition, the DGT can be used to measure pollutants in sediments and soils. The design of the DGT is flexible and work to replace the generic Chelex-100 receiving phase with a number of bespoke resins suited for specific analytes was described. For example, a titanium dioxide layer has been shown to have a good affinity for the sequestration of low levels of uranium in a range of environmental waters. Workers in Japan replaced the chelating resin disk with a special Empore ${ }^{\mathrm{TM}}$ Rad caesium disk in the Chemcatcher and used the device for monitoring radio-caesium $\left({ }^{137} \mathrm{Cs}\right)$ in contaminated field sites around the Fukushima nuclear reactor plant. Preliminary results with the new sampler were encouraging and gave comparable values of ${ }^{137} \mathrm{Cs}$ to those found in concentrated extracts obtained from large volume spot water samples. However, the overall sample preparation time was significantly reduced as counting measurements were undertaken directly off the disk. There was also less risk of exposing laboratory staff to low level radiation during sample processing.

Although the use of passive samplers for measuring concentrations of metals and some nutrients is unequivocal, often workers have given little attention to the effects that water chemistry and method of field deployment may have on results. Information on using these types of sampler in large long-term monitoring campaigns is still quite sparse in comparison to devices used for non-polar substances. How the ambient water quality affects the availability of a given metal for uptake into a 
sampler is complex and needs to be taken into account if the technology is to be used with confidence in a routine regulatory setting. For example: across the seasons, water flow-rate, temperature, $\mathrm{pH}$ and amount of suspended and dissolved particulate matter and nutrients (and hence the propensity for bio-fouling of the diffusional surface) will vary significantly. Each factor affects the distribution of a metal in the water column and hence availability of uptake. The design of the apparatus used to deploy any sampler also has an impact on uptake kinetics. Although in most cases the field location dictates the type of equipment that can be employed, often little consideration is given to this aspect by end users where a range of different kit is utilised in a given monitoring campaign.

\section{Use of passive sampling devices in regulatory monitoring programmes}

It is evident that there is worldwide interest in the use of passive samplers for environmental monitoring. This was not the case 10 years ago when most end users had to be convinced of applicability and reliability of the technology. The recent resurgence of interest in Europe has, in part, been driven by the revised water quality legislation (i.e. WFD in 2001 and the MSFD in 2008 introduced across the Community). A number of large research and demonstration projects funded by the Commission have shown the potential of passive samplers, used in conjunction with other techniques, for monitoring water quality within a regulatory framework. A recent change to the WFD illustrates this point. The updated Directive 2013/39/EU on priority substances with respect to Community water policy introduced very low environmental quality standards (EQSs) for several compounds in surface waters ${ }^{14}$ (e.g. 8-80 pg/L for cypermethrin, 60-600 pg/L for dichlorvos, 32-1,300 pg/L for heptachlor/heptachlorepoxide and 130-650 pg/L for PFOS). This means using lowvolume spot samples of water combined with conventional laboratory analysis will result in method quantification limits higher than the respective EQS. Such methods will not be accepted by the Commission for compliance monitoring within the Directive. An option is the use of passive samplers for in situ extraction of such pollutants from water. Many samplers have high uptake rates (from hundreds of $\mathrm{mL}$ to several L/day), so this may be an option to measure very low concentrations in the field. Moreover, measurement of the free dissolved concentration in water using passive samplers provides a better assessment of exposure of aquatic organisms to 
priority pollutants than whole water sampling. For example, more than $90 \%$ of the compounds identified using a combination of different passive samplers in a trial undertaken by the Environment Agency of England and Wales in 2011 were not identified using routine spot sampling techniques. Many of the substances identified by passive sampling were priority hazardous substances listed in Annex $X$ of the WFD. A similar approach may be needed for fulfilling the future requirements of MSFD. In coastal and marine waters the concentration of most pollutants is generally much lower than those found in surface water due to significant dilution effects. The measurement of such low concentrations by conventional water techniques in these environments will prove challenging.

Nevertheless, passive sampling is not yet applied in regulatory compliance monitoring as the EQSs are not defined for the compartments sampled by this method, e.g. the freely dissolved concentration of a pollutant in the water column. In July 2013, the Network of reference laboratories for monitoring emerging environmental pollutants (NORMAN Association - www.norman-network.net) organised an expert group meeting to bring together eco-toxicologists and experts on monitoring to investigate how the EQS defined for various pollutants could be related to their respective concentrations measured using passive sampling devices - or should the Commission reconsider how EQS are derived? The conclusions are to be disseminated in a position document clarifying where passive sampling fits into the schemes that are currently applied for assessment of the chemical and ecological status of water bodies under the WFD.

Another revision within Directive 2013/39/EU was the opportunity for Member States to use matrices (e.g. biota or sediment) other than water for monitoring very bioaccumulative compounds; provided they could supply evidence that an equal level of protection of aquatic life was being achieved. For these chemicals, biota is the preference for chemical monitoring and the Directive sets out EQS for this matrix. Concentrations of pollutants in biota are related to their concentrations in the aqueous phase. Use of organisms for chemical monitoring, however, introduces natural variability (caused by variable size, age, sex and physiological conditions of sampled organisms) into reported data, which complicates or in some cases precludes their spatial and temporal comparability. Moreover, the specific biota 
species required for chemical monitoring may not be available at some sampling sites. A potential solution is to apply abiotic passive sampling methods that provide "biomimetic" pollutant measurements, i.e. simulate the bio-concentration of pollutants from water into aquatic organisms, with a low inherent variability. Partition-based samplers equilibrated with water or sediment can be used to estimate lipid normalised concentrations of pollutants in aquatic organisms in the monitored system, providing the relevant lipid/polymer partition coefficients are available. Another application is based on direct equilibration of polymer-based passive samplers with biota tissue. The equilibrium concentrations obtained in tissue enable a direct comparison of contaminant levels between organisms, species or trophic levels when studying bio-magnification.

Within this topic area an update of the inter-laboratory study on the use of passive samplers for monitoring of emerging pollutants organised in 2011 by the NORMAN Association together with the European DG Joint Research Centre was given ${ }^{5}$. Study participants were free to apply passive samplers that they use routinely in their laboratories. In addition, organisers provided silicone rubber (for non-polar compounds) and POCIS (for polar compounds) samplers to be analysed in all participant laboratories. The exercise showed that the within laboratory precision obtained from use of the samplers was mostly satisfactory, but the laboratory analysis was in most cases the main source of between laboratory variability. The commonly used liquid chromatography/mass spectrometry technique is very susceptible to matrix effects, especially when using electrospray ionisation ${ }^{15}$. These effects include enhancement of ionisation as well as suppression. Extensive cleanup of extracts from samplers may be required to produce data that is fit for purpose. It is clear, however, for future successful application of these devices in monitoring campaigns the variability that originates from laboratory analysis must be minimised. This will require training of laboratories in routine preparation and analysis of extracts from samplers as well as organisation of proficiency testing schemes. The final report from the study is in preparation. In parallel, there must also be knowledge of how to interpret information obtained from passive samplers, particularly in the area of uncertainty of data. 
The presentations at IPSW 2013 showed some of the key developments taking place in the area of passive sampling, with a key focus on monitoring of water quality. Some areas where polymeric devices can be used to assist regulators meet the new EQS for a wide range of priority substances within the latest revision of the WFD showed the future potential of this monitoring approach. Several challenges still remain, particularly for measuring polar pollutants and further research is needed here. The work of the NORMAN Association is doing much to disseminate the potential of the technology that is now being taken up by an ever increasing number of end users.

\section{References}

1. R. W. Coutant, R. G. Lewis and J. Mulik, Anal. Chem., 1985, 57, 219-223.

2. European Commission, The European Water Framework Directive (WFD; 2000/60/EC)

(http://eur-lex.europa.eu/LexUriServ/LexUriServ.do?uri=OJ:L:2000:327:0001:0072:EN:PDF)

3. European Commission, The European Marine Strategy Framework Directive (MSFD; 2008/56/EC)

(http://eur-lex.europa.eu/LexUriServ/LexUriServ.do?uri=OJ:L:2008:164:0019:0040:EN:PDF)

4. I. J. Allan, B. Vrana, R. Greenwood, G. A. Mills, J. Knutsson, A. Holmberg, N. Guigues, A.-M. Fouillac and S. Laschi, TrAC, Trends Anal. Chem., 2006, 25, 704-715.

5. G. A. Mills, R. Greenwood, B. Vrana, I. Allan T. and Ocelka, J. Environ. Monit., 2011, 13, 2979-2982.

6. J. N. Huckins, M. W. Tubergen and G. K. Manuweera, Chemosphere, 1990, 20, 533-552.

7. K. Yates, I. Davies, L. Webster, P. Pollard, L. Lawton and C. Moffat, J. Environ. Monit., 2007, 9, 1116-1121. 
8. D. A. Alvarez, J. D. Petty, J. N. Huckins, T. L. Jones-Lepp, D. T. Getting, J. P. Goddard, et al., Environ. Toxicol. Chem., 2004, 23, 1640-1648.

9. N. Morin, C. Miege, J. Randon and M. Coquery, TrAC, Trends Anal. Chem., 2012, 36, 144-175.

10. N. Mazzella, S. Lissalde, S. Moreira, F. Delmas, P. Mazellier and J. N. Huckins, Environ. Sci. Technol., 2010, 44, 1713-1719.

11. C.-E. Chen, H. Zhang and K. C. Jones, J. Environ. Monit., 2012,14, 1523-1530.

12. E. Bailly, Y. Levi and S. Karolak, Environ. Pollut., 2013, 174, 100-105.

13. I. J. Allan, J. Knutsson, N. Guigues, G. A. Mills, A.-M. Fouillac and R. Greenwood, J. Environ. Monit., 2007, 9, 672-681.

14. European Commission, Directive 2013/39/EU amending Directives 2000/60/EC and 2008/105/EC as regards priority substances in the field of water policy. (http://eur-lex.europa.eu/LexUriServ/LexUriServ.do?uri=OJ:L:2013:226:0001:0017:EN:PDF)

15. J. E. Renew and C.-H. Huang, J. Chromatogr. A, 2004, 1042, 113-121.

\section{Graham A. Mills}

University of Portsmouth, Portsmouth, UK

Anthony Gravell

Natural Resources Wales, Llanelli, UK

Branislav Vrana

RECETOX, Masaryk University, Brno, Czech Republic/Water Research Institute, Bratislava, Slovakia

Christopher Harman

Norwegian Institute for Water Research (NIVA), Oslo, Norway

Hélène Budzinski 
Université Bordeaux, Bordeaux, France

Nicolas Mazzella

IRSTEA, Cestas, France

Tomáš Ocelka

Institute of Public Health Ostrava, Ostrava, Czech Republic 